\title{
An Improved Bound on the Number of Unit Area Triangles
}

\author{
Roel Apfelbaum • Micha Sharir
}

Received: 12 May 2009 / Revised: 11 February 2010 / Accepted: 9 May 2010 /

Published online: 15 June 2010

(C) Springer Science+Business Media, LLC 2010

\begin{abstract}
We show that the number of unit-area triangles determined by a set of $n$ points in the plane is $O\left(n^{9 / 4+\varepsilon}\right)$, for any $\varepsilon>0$, improving the recent bound $O\left(n^{44 / 19}\right)$ of Dumitrescu et al.
\end{abstract}

Keywords Geometric incidences · Repeated configurations · Unit area triangles · Cutting

\section{Introduction}

In 1967, A. Oppenheim (see [6]) asked the following question: Given $n$ points in the plane and $A>0$, how many triangles spanned by the points can have area $A$ ? By applying a scaling transformation, one may assume that $A=1$ and count the triangles of unit area. Erdôs and Purdy [5] showed that a $\sqrt{\log n} \times(n / \sqrt{\log n})$ section of the integer lattice determines $\Omega\left(n^{2} \log \log n\right)$ triangles of the same area. They also showed that the maximum number of such triangles is at most $O\left(n^{5 / 2}\right)$. In 1992 , Pach and Sharir [8] improved the bound to $O\left(n^{7 / 3}\right)$, using the Szemerédi-Trotter theorem [11] on the number of point-line incidences. Recently, Dumitrescu et al. [4] have further improved the upper bound to $O\left(n^{44 / 19}\right)=O\left(n^{2.3158}\right)$, by estimating the number of incidences between the given points and a 4-parameter family of quadratic curves.

Work on this paper was supported by NSF Grants CCF-05-14079 and CCF-08-30272, by a grant from the US-Israeli Binational Science Foundation, by grant 155/05 from the Israel Science Fund, and by the Hermann Minkowski-MINERVA Center for Geometry at Tel Aviv University.

R. Apfelbaum ( $\varangle) \cdot$ M. Sharir

School of Computer Science, Tel Aviv University, Tel Aviv 69978, Israel e-mail: roel6@hotmail.com

M. Sharir

Courant Institute of Mathematical Sciences, New York University, New York, NY 10012, USA

e-mail: michas@post.tau.ac.il 
In this paper we further improve the bound to $O\left(n^{9 / 4+\varepsilon}\right)$ for any $\varepsilon>0$. Our proof borrows some ideas from [4] but works them into a different approach, which reduces the problem to bounding the number of incidences between points and certain kind of surfaces in three dimensions.

\section{Unit-Area Triangles in the Plane}

To simplify the notation, we write $O^{*}(f(n))$ for an upper bound of the form $C_{\varepsilon} f(n)$. $n^{\varepsilon}$, which holds for any $\varepsilon>0$, where the constant of proportionality $C_{\varepsilon}$ depends on $\varepsilon$.

Theorem 2.1 The number of unit-area triangles spanned by $n$ points in the plane is $O^{*}\left(n^{9 / 4}\right)$.

Proof We begin by borrowing some notation and preliminary ideas from [4]. Let $S$ be a given set of $n$ points in the plane. Consider a triangle $\Delta=\Delta a b c$ spanned by $S$. We call the three lines containing the three sides of $\Delta a b c$, base lines of $\Delta$, and the three lines parallel to the base lines and incident to the respective third vertices, top lines of $\Delta$.

For a parameter $k, 1 \leq k \leq \sqrt{n}$, to be optimized later, call a line $\ell k$-rich (resp., $k$-poor) if $\ell$ contains at least $k$ (resp., fewer than $k$ ) points of $S$. Call a triangle $\Delta a b c$ $k$-rich if each of its three top lines is $k$-rich; otherwise $\Delta$ is $k$-poor.

We first observe that the number of $k$-poor unit-area triangles spanned by $S$ is $O\left(n^{2} k\right)$. Indeed, assign a $k$-poor unit-area triangle $\Delta a b c$ whose top line through $c$, say, is $k$-poor to the opposite base $a b$. Then all the triangles assigned to a base $a b$ are such that their third vertex lies on one of the two lines parallel to $a b$ at distance $2 /|a b|$, where that line contains fewer than $k$ points of $S$. Hence, a base $a b$ can be assigned at most $2 k$ triangles, and the bound follows.

So far, the analysis follows that of [4]. We now focus the analysis on the set of $k$-rich unit-area triangles spanned by $S$ and use a different approach.

Let $L$ denote the set of $k$-rich lines, and let $Q$ denote the set of all pairs

$$
\{(\ell, p) \mid \ell \in L, p \in S \cap \ell\} .
$$

By the Szemerédi-Trotter theorem [11], we have, for any $k \leq \sqrt{n}, m:=|L|=$ $O\left(n^{2} / k^{3}\right)$ and $N:=|Q|=O\left(n^{2} / k^{2}\right)$.

A pair $\left(\ell_{1}, p_{1}\right),\left(\ell_{2}, p_{2}\right)$ of elements of $Q$ is said to match if the triangle with vertices $p_{1}, p_{2}, \ell_{1} \cap \ell_{2}$ has area 1 ; see Fig. 1 .

To upper bound the number of unit-area triangles, all of whose three top lines are $k$-rich, it suffices to bound the number of matching pairs in $Q$. Indeed, given such a unit-area triangle $\Delta p_{1} p_{2} q$, let $\ell_{1}$ (resp., $\ell_{2}$ ) be the top line of $\Delta p_{1} p_{2} q$ through $p_{1}$ (resp., through $\left.p_{2}\right)$. Then $\left(\ell_{1}, p_{1}\right)$ and $\left(\ell_{2}, p_{2}\right)$ form a matching pair in $Q$, by definition (again, see Fig. 1). Conversely, a matching pair $\left(\ell_{1}, p_{1}\right),\left(\ell_{2}, p_{2}\right)$ determines at most one unit-area triangle $p_{1} p_{2} q$, where $q$ is the intersection point of the line through $p_{1}$ parallel to $\ell_{2}$ and the line through $p_{2}$ parallel to $\ell_{1}$; we get an actual triangle if and only if the point $q$ belongs to $S$. 
Fig. 1 The ordered pair $\left(\left(\ell_{1}, p_{1}\right),\left(\ell_{2}, p_{2}\right)\right)$ is a matching pair of elements of $Q$

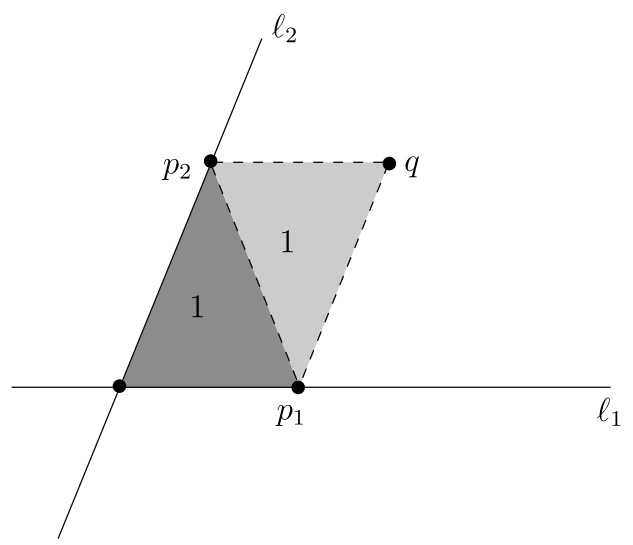

In other words, our problem is now reduced to that of bounding the number of matching pairs in $Q$. (Since we do not enforce the condition that the third point $q$ of the corresponding triangle belongs to $S$, we most likely over-estimate the true bound.)

Since elements of $Q$ have three degrees of freedom, we can represent them in an appropriate three-dimensional parametric space. For example, we can assume that no line in $L$ is vertical, and parameterize an element $(\ell, p)$ of $Q$ by the triple $(a, b, \kappa)$, where $(a, b)$ are the coordinates of $p$, and $\kappa$ is the slope of $\ell$. For simplicity of notation, we refer to this three-dimensional parametric space as $\mathbb{R}^{3}$.

So far, the matching relationship is symmetric. To simplify the analysis, and with no loss of generality, we make it asymmetric, by requiring that, in an (ordered) matching pair $\left(\ell_{1}, p_{1}\right),\left(\ell_{2}, p_{2}\right), \overrightarrow{o p_{2}}$ lies counterclockwise to $\overrightarrow{o p_{1}}$, where $o=\ell_{1} \cap \ell_{2}$. See Fig. 1.

Let us express the matching condition algebraically. Let $(a, b, \kappa) \in \mathbb{R}^{3}$ be the triple representing a pair $(\ell, p)$, and $(x, y, w) \in \mathbb{R}^{3}$ be the triple representing another pair $\left(\ell^{\prime}, p^{\prime}\right)$. Clearly, $w \neq \kappa$ in a matching pair. The lines $\ell$ and $\ell^{\prime}$ intersect at a point $o$, for which there exist real parameters $t, s$ which satisfy $o=(a+t, b+\kappa t)=(x+$ $s, y+w s)$, or

$$
t=\frac{y-b-w(x-a)}{\kappa-w}, \quad s=\frac{y-b-\kappa(x-a)}{\kappa-w} .
$$

It is now easy to verify that the condition of matching, with $\overrightarrow{o p}^{\prime}$ lying counterclockwise to $\overrightarrow{o p}$, is given by

$$
(y-b-\kappa(x-a))(y-b-w(x-a))=2(w-\kappa) \quad \text { and } \quad w \neq \kappa,
$$

or, alternatively,

$$
w=\frac{(y-b-\kappa(x-a) r)(y-b)+2 \kappa}{(y-b-\kappa(x-a))(x-a)+2} \quad \text { and } \quad w \neq \kappa .
$$


Similarly, the condition of "reverse" matching, with $\overrightarrow{o p}$ ' lying clockwise to $\overrightarrow{o p}$, is given by

$$
w=\frac{(y-b-\kappa(x-a))(y-b)-2 \kappa}{(y-b-\kappa(x-a))(x-a)-2} \quad \text { and } \quad w \neq \kappa .
$$

Fix an element $(\ell, p)$ of $Q$ and associate with it a surface $\sigma_{\ell, p} \subset \mathbb{R}^{3}$ which is the locus of all pairs $\left(\ell^{\prime}, p^{\prime}\right)$ that match $(\ell, p)$ (i.e., $(\ell, p),\left(\ell^{\prime}, p^{\prime}\right)$ is an ordered matching pair). By the preceding analysis, $\sigma_{\ell, p}$ satisfies $(1)$, where $(a, b, \kappa)$ is the parameterization of $(\ell, p)$ and is thus a two-dimensional algebraic surface in $\mathbb{R}^{3}$ of degree 3 . We thus obtain a system $\Sigma$ of $N$ two-dimensional algebraic surfaces in $\mathbb{R}^{3}$, and a set $Q$ of $N$ points in $\mathbb{R}^{3}$, and our goal is to bound the number of incidences between $Q$ and $\Sigma$.

The main technical step in the analysis is to rule out the possible existence of degeneracies in the incidence structure, where many points are incident to many surfaces; this might happen when many points lie on a common intersection curve of many surfaces (a situation which might arise, e.g., in the case of planes and points in $\mathbb{R}^{3}$ ). However, for the class of surfaces under consideration, namely, the surfaces $\sigma_{\ell, p}$ generated by some line-point incidence pair $(\ell, p)$, such a degeneracy is impossible, as the following lemma shows.

Lemma 2.2 Let $\left(\ell_{1}, p_{1}\right)$ and $\left(\ell_{2}, p_{2}\right)$ be two distinct line-point incidence pairs, let $\gamma=\sigma_{\ell_{1}, p_{1}} \cap \sigma_{\ell_{2}, p_{2}}$ be the intersection curve of their associated surfaces, and assume that $\gamma$ is nonempty. Let $(\ell, p)$ be some incidence pair and assume further that $\sigma_{\ell, p} \supset$ $\gamma$. Then either $(\ell, p)=\left(\ell_{1}, p_{1}\right)$ or $(\ell, p)=\left(\ell_{2}, p_{2}\right)$.

Proof We establish the equivalent claim that, given a curve $\gamma$ which is the intersection of some unknown pair of surfaces $\sigma_{\ell_{1}, p_{1}}$ and $\sigma_{\ell_{2}, p_{2}}$, one can reconstruct $\left(\ell_{1}, p_{1}\right)$ and $\left(\ell_{2}, p_{2}\right)$ uniquely (up to a swap between the two incidence pairs) from $\gamma$. Moreover, it is enough to know the projection $\gamma^{*}$ of $\gamma$ onto the $x y$-plane in order to uniquely reconstruct the incidence pairs $\left(\ell_{1}, p_{1}\right)$ and $\left(\ell_{2}, p_{2}\right)$ that generate $\gamma$.

We start by computing the algebraic representation of $\gamma^{*}$. Let $\left(a_{1}, b_{1}, \kappa_{1}\right)$ and $\left(a_{2}, b_{2}, \kappa_{2}\right)$ be the respective parameterizations of $\left(\ell_{1}, p_{1}\right)$ and $\left(\ell_{2}, p_{2}\right)$. By $(1), \gamma^{*}$ satisfies the equation

$$
\frac{\left(y-b_{1}-\kappa_{1}\left(x-a_{1}\right)\right)\left(y-b_{1}\right)+2 \kappa_{1}}{\left(y-b_{1}-\kappa_{1}\left(x-a_{1}\right)\right)\left(x-a_{1}\right)+2}=\frac{\left(y-b_{2}-\kappa_{2}\left(x-a_{2}\right)\right)\left(y-b_{2}\right)+2 \kappa_{2}}{\left(y-b_{2}-\kappa_{2}\left(x-a_{2}\right)\right)\left(x-a_{2}\right)+2} .
$$

Recall the additional requirement in (1), namely that $w \neq \kappa_{1}$ and $w \neq \kappa_{2}$. This requirement is implicit in (1) and in (3), meaning that (3) is defined only for values of $x$ and $y$ for which the value of $w$ is not $\kappa_{1}$ or $\kappa_{2}$. Consulting (1), this implies that no point $(x, y) \in \gamma$ can satisfy $y-b_{1}=\kappa_{1}\left(x-a_{1}\right)$ or $y-b_{2}=\kappa_{2}\left(x-a_{2}\right)$. Put

$$
L_{1}=y-b_{1}-\kappa_{1}\left(x-a_{1}\right) \quad \text { and } \quad L_{2}=y-b_{2}-\kappa_{2}\left(x-a_{2}\right)
$$

and write (3) as

$$
\frac{L_{1}\left(y-b_{1}\right)+2 \kappa_{1}}{L_{1}\left(x-a_{1}\right)+2}=\frac{L_{2}\left(y-b_{2}\right)+2 \kappa_{2}}{L_{2}\left(x-a_{2}\right)+2}
$$




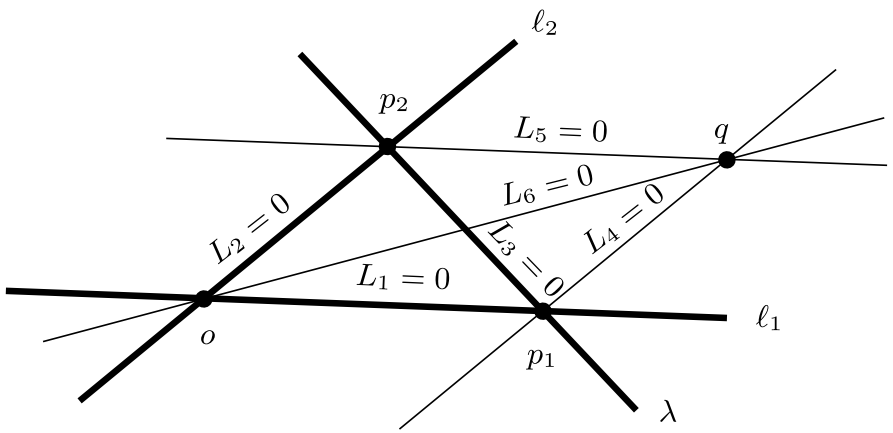

Fig. 2 The lines $L_{i}=0$ for $i=1, \ldots, 6$ in the general case. The line $L_{3}=0$ connects $p_{1}$ and $p_{2}, L_{4}=0$ passes through $p_{1}$ and is parallel to $\ell_{2}, L_{5}=0$ passes through $p_{2}$ and is parallel to $\ell_{1}$, and $L_{6}=0$ connects $o=\ell_{1} \cap \ell_{2}$ with the intersection point $q$ of $L_{4}=0$ and $L_{5}=0$ (and bisects the edge $p_{1} p_{2}$ )

or

$$
\left(L_{1}\left(y-b_{1}\right)+2 \kappa_{1}\right)\left(L_{2}\left(x-a_{2}\right)+2\right)=\left(L_{2}\left(y-b_{2}\right)+2 \kappa_{2}\right)\left(L_{1}\left(x-a_{1}\right)+2\right),
$$

which we can rewrite as

$$
L_{1} L_{2} L_{3}+2 L_{6}+4 C=0 \text { and } L_{1} \neq 0, L_{2} \neq 0
$$

where

$$
\begin{aligned}
& L_{3}=\left(b_{2}-b_{1}\right) x-\left(a_{2}-a_{1}\right) y+\left(a_{2} b_{1}-a_{1} b_{2}\right), \\
& L_{4}=y-b_{1}-\kappa_{2}\left(x-a_{1}\right), \\
& L_{5}=y-b_{2}-\kappa_{1}\left(x-a_{2}\right), \\
& L_{6}=L_{1} L_{4}-L_{2} L_{5} \\
& C=\kappa_{1}-\kappa_{2}
\end{aligned}
$$

it is easily verified that $L_{6}$ is also a linear expression is $x, y$. Figure 2 illustrates the different lines defined by the linear equations $L_{i}=0$, and their relations with $\left(\ell_{1}, p_{1}\right)$ and $\left(\ell_{2}, p_{2}\right)$. The linearity of $L_{1}, L_{2}, L_{3}$, and $L_{6}$ implies that (4) of $\gamma^{*}$ is cubic.

We have the following two special cases to rule out:

1. If $p_{1}=p_{2}$, that is, $a_{1}=a_{2}$ and $b_{1}=b_{2}$, then $L_{3}=0, L_{4}=L_{2}$, and $L_{5}=L_{1}$. But then the equation becomes $4 C=0$, or $\kappa_{1}=\kappa_{2}$, contrary to the assumption that $\left(\ell_{1}, p_{1}\right) \neq\left(\ell_{2}, p_{2}\right)$. Hence, the equation has no solutions, meaning that $\gamma$ is empty and the surfaces do not intersect.

2. If $\ell_{1}=\ell_{2}$ but $p_{1} \neq p_{2}$, that is, $\kappa_{1}=\kappa_{2}=\left(b_{2}-b_{1}\right) /\left(a_{2}-a_{1}\right)$, then $L_{1}=L_{2}=$ $L_{4}=L_{5}, L_{3}=\left(a_{1}-a_{2}\right) L_{1}$, and $C=0$, resulting in the equation $\left(L_{1}\right)^{3}=0$, which is not allowed in (4). Hence $\gamma$ is not defined in this case either.

We can therefore restrict our attention to the general case. Consider the cubic part of the equation $L_{1} L_{2} L_{3}$. In this term, each factor can be thought of as a line defined 
by the equation $L_{i}=0$ for $i=1,2,3$. The lines $L_{1}=0$ and $L_{2}=0$ respectively are simply $\ell_{1}$ and $\ell_{2}$, whereas $L_{3}=0$ is the line $\lambda$ passing through $p_{1}$ and $p_{2}$ (see Fig. 2). Note that $\lambda$ may coincide with one of the other two lines. Indeed, if $p_{1}$ happens to be incident with $\ell_{2}$, then $\lambda$ coincides with $\ell_{2}$. Similarly, if $p_{2} \in \ell_{1}$, then $\lambda$ coincides with $\ell_{1}$ (these are the only possible coincidences, since we have ruled out the case $\ell_{1}=\ell_{2}$ ). These cases will be handled shortly, but for now, we ignore them and consider the general case. In this case, $\gamma^{*}$ has three distinct asymptotes given by $L_{1}=0$, $L_{2}=0$, and $L_{3}=0$; see Lemma A.2 in the Appendix.

Using this fact, one can reconstruct the two line-point pairs that generate $\gamma^{*}$ as follows. Suppose we are given a curve $\gamma^{*}$ generated by some unknown pair of incidence pairs, $\left(\ell_{1}, p_{1}\right)$ and $\left(\ell_{2}, p_{2}\right)$, and we want to reconstruct these pairs. $\gamma^{*}$ is given as the zero set of some cubic bivariate polynomial $f(x, y)=0$, where $f$ can be written as $f(x, y)=c\left(L_{1} L_{2} L_{3}+2 L_{6}+4 C\right)$, but the decomposition of $f$ into $L_{1}, L_{2}, L_{3}, L_{6}, C$, and $c$ is unknown, and, moreover, is not known a priori to be unique (a fact which we establish in this proof). First, we find its three asymptotes $\Lambda_{1}=0, \Lambda_{2}=0$, and $\Lambda_{3}=0$, where for each $i=1,2,3, \Lambda_{i}$ is linear in $x$ and $y$. Since, by Lemma A.2, these asymptotes are $L_{1}=0, L_{2}=0$, and $L_{3}=0$, we know that each $\Lambda_{i}$ is equal to some $L_{j}$ multiplied by a constant, but we do not know which is which. To determine the roles of the asymptotes correctly, observe that $\Lambda_{1} \Lambda_{2} \Lambda_{3}=\mu L_{1} L_{2} L_{3}$ for some constant $\mu$. Thus, there exists some unique constant $v$ such that $f(x, y)-v \Lambda_{1} \Lambda_{2} \Lambda_{3}=\Lambda_{4}$ is linear in $x$ and $y$. The line $\Lambda_{4}=0$ is parallel to the line $L_{6}=0$, which happens to be the median of the triangle spanned by the three asymptotes, which emanates from the vertex $o=\ell_{1} \cap \ell_{2}$ and bisects the edge $p_{1} p_{2}$; see Fig. 2. We thus have enough information to determine which vertex of the triangle is $o$, which are $p_{1}$ and $p_{2}$, and which edges of the triangle are supported by $\ell_{1}$ and $\ell_{2}$. This proves the lemma for the general case where all the points and lines are distinct, and no point lies on both lines $\ell_{2}, \ell_{2}$.

Finally, consider the case where $p_{2} \in \ell_{1}$ (a symmetric argument applies when $\left.p_{1} \in \ell_{2}\right)$. In this case, $L_{1}=L_{5}$ and $L_{3}=\left(a_{1}-a_{2}\right) L_{1}$, so the equation of the curve $\gamma^{*}$ can be rewritten as

$$
\left(a_{1}-a_{2}\right) L_{1}^{2} L_{2}+2 L_{1}\left(L_{4}-L_{2}\right)+4 C=0 .
$$

Note that $a_{1} \neq a_{2}$ under the preliminary assumption that there are no vertical lines in the system, since both $p_{1}=\left(a_{1}, b_{1}\right)$ and $p_{2}=\left(a_{2}, b_{2}\right)$ are on $\ell_{1}$. Note also that $C=$ $\kappa_{1}-\kappa_{2} \neq 0$, for otherwise, $\ell_{1}$ and $\ell_{2}$ would have to coincide, a case which we have ruled out earlier. Finally, note that $s=L_{4}-L_{2}=b_{2}-b_{1}-\kappa_{2}\left(a_{2}-a_{1}\right)=C\left(a_{2}-a_{1}\right)$ is a nonzero constant. Hence, the equation of $\gamma^{*}$ is, up to a constant multiple,

$$
\left(a_{1}-a_{2}\right) L_{1}^{2} L_{2}+2 s L_{1}+4 C=0 .
$$

This equation defines a cubic curve with two asymptotes given by $L_{1}=0$ and $L_{2}=0$, namely, the lines $\ell_{1}$ and $\ell_{2}$; see Lemma A.3 in the Appendix. Since $C \neq 0$, it follows that $\gamma^{*}$ does not intersect $L_{1}=0$, whereas $L_{2}=0$ is intersected at a single point $(x, y)$ for which $L_{1}=2 /\left(a_{1}-a_{2}\right)$. Using this point, one can compute the values of $\left(a_{1}-a_{2}\right), C$, and $s$ and hence, reconstruct the line $L_{4}=0$. The point $p_{1}$ is then simply the intersection of the lines $L_{1}=0$ and $L_{4}=0$. Thus, one can uniquely reconstruct $\ell_{1}, \ell_{2}, p_{1}$, and $p_{2}$ in this case too. This completes the proof of Lemma 2.2. 
Bounding the Number of Incidences: A Simple Bound Lemma 2.2 implies that the incidence graph between $\Sigma$ and $Q$ does not contain $K_{3,10}$ as a subgraph, or, in other words, no three distinct surfaces of $\Sigma$ and ten distinct points of $Q$ can all be incident to one another. Indeed, the intersection points of three surfaces $\sigma_{\ell_{i}, p_{i}}$, $i=1,2,3$, are the intersection points of the two curves $\gamma_{1,2}=\sigma_{\ell_{1}, p_{1}} \cap \sigma_{\ell_{2}, p_{2}}$ and $\gamma_{1,3}=\sigma_{\ell_{1}, p_{1}} \cap \sigma_{\ell_{3}, p_{3}}$. These intersection points project to (some of) the intersection points of the projections $\gamma_{1,2}^{*}$ and $\gamma_{1,3}^{*}$ of $\gamma_{1,2}$ and $\gamma_{1,3}$, respectively, onto the $x y$ plane. By Lemma 2.2, these two curves are distinct (or empty). Since each of them is cubic, and since, as shown in Lemmas A.2 and A.3 in the Appendix, they are the zero sets of irreducible polynomials, Bézout's theorem [9] implies that they intersect in at most $3^{2}=9$ points. Hence, the incidence graph between $\Sigma$ and $Q$ does not contain $K_{3,10}$, so by the Kővari-Sós-Turán theorem [7], the number of incidences between $\Sigma$ and $Q$ can be bounded by $O\left(|\Sigma||Q|^{2 / 3}+|Q|\right)$. Since the matching relation is essentially symmetric (up to some sign changes; see (1) and (2)), we can interchange the roles of points and surfaces and conclude that the number of incidences is also at most

$$
O\left(|Q||\Sigma|^{2 / 3}+|\Sigma|\right)
$$

Cutting To improve the bound, we apply the following fairly standard space decomposition technique. Fix a parameter $r$, whose specific value will be chosen later, and construct a $(1 / r)$-cutting $\Xi$ of $\mathcal{A}(\Sigma)$ [2]. We use the more simple-minded technique in which we choose a random sample $R$ of $O(r \log r)$ surfaces of $\Sigma$ and construct the vertical decomposition (see, e.g., $[3,10]$ ) of the arrangement $\mathcal{A}(R)$. We obtain $O^{*}\left(r^{3}\right)$ relatively open cells of dimensions $0,1,2$, and 3 , each of which is crossed by (intersected by, but not contained in) at most $|\Sigma| / r=N / r$ surfaces; this latter property holds with high probability, and we simply assume that our sample $R$ does satisfy it.

Summing Over All Cells Fix a cell $\tau$ of $\Xi$ and put $Q_{\tau}:=Q \cap \tau$ and $m_{\tau}:=\left|Q_{\tau}\right|$. Let $\Sigma_{\tau}$ denote the subset of surfaces of $\Sigma$ which cross $\tau$ and put $N_{\tau}:=\left|\Sigma_{\tau}\right| \leq N / r$.

We now apply the simple bound (6) obtained in the first step to each cell $\tau$ of our cutting $\Xi$, handling, for the time being, only surfaces that cross $\tau$. The overall number of incidences is

$$
\sum_{\tau \in \Xi} O\left(m_{\tau} N_{\tau}^{2 / 3}+N_{\tau}\right)
$$

which, using the bounds $N_{\tau} \leq N / r$ and $\sum_{\tau} m_{\tau}=N$, is

$$
O^{*}\left(N(N / r)^{2 / 3}+N r^{2}\right)=O^{*}\left(N^{5 / 3} / r^{2 / 3}+N r^{2}\right) \text {. }
$$

To minimize this expression, we choose $r=N^{1 / 4}$, making it $O^{*}\left(N^{3 / 2}\right)$.

We also have to take into account incidences between points in a cell $\tau$ and surfaces that fully contain $\tau$. This is done separately for cells of dimension 0,1 , and 2 (it is vacuous for cells of dimension 3 ). Indeed, a two-dimensional cell $\tau$ is contained in exactly one surface, so a point $w \in \tau$ takes part in only one such incidence. Thus, in this case we only need to add $N$, the number of points, to the above bound. 
The same argument applies for points in one-dimensional cells. Assuming that the vertical decomposition is performed in a generic coordinate frame, it suffices to consider only one-dimensional cells that are portions of the intersection curves between the surfaces of $\Sigma$. By Lemma 2.2, each such cell $\tau$ is contained in exactly two surfaces of $\Sigma$. Thus, we need to add at most $2 N$ to the number of incidences to handle these cells.

Each cell of dimension 0 is a single point $w$, and, arguing as above, we may assume it to be a vertex of the undecomposed arrangement $\mathcal{A}(R)$. Any surface $\sigma$ incident to $w$ has to cross or bound an adjacent full-dimensional cell $\tau^{*}$, so we charge the incidence of $\sigma$ with $w$ to the pair $\left(\tau^{*}, \sigma\right)$ and note that such a pair can be charged only $O(1)$ times. It follows that the number of incidences with zero-dimensional cells of $\Xi$ is $O^{*}\left(r^{3}+r^{3}(N / r)\right)=O^{*}\left(r^{2} N\right)$, which, for the chosen value of $r$, is equal to the bound obtained above for the crossing surfaces.

In conclusion, the overall number of incidences between $\Sigma$ and $Q$ is $O^{*}\left(N^{3 / 2}\right)$.

Recall now that $N=O\left(n^{2} / k^{2}\right)$ and that we also have the bound $O\left(n^{2} k\right)$ for the number of unit-area triangles with at least one $k$-poor top line. Thus, the overall bound on the number of unit-area triangles is

$$
O^{*}\left(\frac{n^{3}}{k^{3}}+n^{2} k\right),
$$

which, if we choose $k=n^{1 / 4}$, becomes $O^{*}\left(n^{9 / 4}\right)$, as asserted.

\section{Appendix: Asymptotes of Cubic Curves}

In this appendix, we state, without proofs, several properties of the cubic curves defined by (4) and (5) of Sect. 2, which are needed for our analysis. The proofs are simple and elementary and are omitted in this version. They can be found in a somewhat fuller version of the paper [1].

Lemma A.1 Let $\lambda_{1}$ and $\lambda_{2}$ be two distinct lines in $\mathbb{R}^{2}$ given by the equations $\Lambda_{i}=0$, where $\Lambda_{i}=\alpha_{i} x+\beta_{i} y+\gamma_{i}$, and $\alpha_{i}$ and $\beta_{i}$ are both nonzero for $i=1,2$. Let $f(x, y)$ be the bivariate cubic polynomial $f(x, y)=x y \Lambda_{1}+\Lambda_{2}$. Then (a) $f(x, y)$ is irreducible, and (b) its zero set $f(x, y)=0$ is asymptotic to the $x$-axis and to the $y$-axis.

Lemma A.2 Let $\ell_{1}, \ldots, \ell_{4}$ be four distinct lines in $\mathbb{R}^{2}$ given by the equations $L_{i}=0$, where $L_{i}=A_{i} x+B_{i} y+C_{i}$ for $i=1, \ldots, 4$. Assume that no pair of $\ell_{1}, \ell_{2}, \ell_{3}$ is parallel and that $\ell_{4}$ is not parallel to any of $\ell_{1}$ and $\ell_{2}$. Put $f(x, y)=L_{1} L_{2} L_{3}+L_{4}$, and let $\Gamma$ be the algebraic cubic curve $f(x, y)=0$. Then $f$ is irreducible, and $\Gamma$ is asymptotic to the lines $\ell_{1}, \ell_{2}, \ell_{3}$.

Lemma A.3 Let $\ell_{1}$ and $\ell_{2}$ be two distinct intersecting lines in $\mathbb{R}^{2}$ given by the equations $L_{i}=0$, where $L_{i}=A_{i} x+B_{i} y+C_{i}$ for $i=1,2$. Put $f(x, y)=L_{1}^{2} L_{2}+L_{1}+C$ for some constant $C$, and let $\Gamma$ be the algebraic curve defined by $f(x, y)=0$. Then $\Gamma$ is asymptotic to the lines $\ell_{1}$ and $\ell_{2}$. Furthermore, if $C \neq 0$, then $f$ is an irreducible bivariate polynomial. 


\section{References}

1. Apfelbaum, R., Sharir, M.: An improved bound on the number of unit area triangles. arXiv:1001.4764

2. Chazelle, B.: Cuttings. In: Mehta, D., Sahni, S. (eds.): Handbook of Data Structures and Applications. Chapman and Hall/CRC Press, New York (2005), Chap. 25

3. Clarkson, K., Edelsbrunner, H., Guibas, L., Sharir, M., Welzl, E.: Combinatorial complexity bounds for arrangements of curves and spheres. Discrete Comput. Geom. 5, 99-160 (1990)

4. Dumitrescu, A., Sharir, M., Tóth, Cs.D.: Extremal problems on triangle areas in two and three dimensions. J. Comb. Theory, Ser. A 116, 1177-1198 (2009)

5. Erdős, P., Purdy, G.: Some extremal problems in geometry. J. Comb. Theory 10, 246-252 (1971)

6. Erdős, P., Purdy, G.: Extremal problems in combinatorial geometry. In: Graham, R., Grötschel, M., Lovász, L. (eds.): Handbook of Combinatorics, vol. 1, pp. 809-874. Elsevier, Amsterdam (1995)

7. Kővari, T., Sós, V.T., Turán, P.: On a problem of K. Zarankiewicz. Colloq. Math. 3, 50-57 (1954)

8. Pach, J., Sharir, M.: Repeated angles in the plane and related problems. J. Comb. Theory, Ser. A 59, 12-22 (1992)

9. Shafarevich, I.R.: Basic Algebraic Geometry. Springer, Berlin (1977)

10. Sharir, M., Agarwal, P.K.: Davenport-Schinzel Sequences and Their Geometric Applications. Cambridge University Press, New York (1995)

11. Szemerédi, E., Trotter, W.T.: Extremal problems in discrete geometry. Combinatorica 3, 381-392 (1983) 